\title{
USE OF A SIMULATOR TO OBJECTIVELY DISTINGUISH BEHAVIORS BETWEEN LOW-RISK AND HIGH-RISK DRIVERS
}

\author{
Yi-Ching Lee ${ }^{1}$, Noelle LaVoie ${ }^{2}$, Ursula Lauper ${ }^{2}$, \& Anna Cianciolo $^{3}$ \\ ${ }^{1}$ Center for Injury Research and Prevention, Children’s Hospital of Philadelphia \\ Philadelphia, Pennsylvania, USA \\ ${ }^{2}$ Parallel Consulting, San Francisco, California, USA \\ ${ }^{3}$ Command Performance Research, Champaign, Illinois, USA \\ Email: LeeY1@email.chop.edu
}

\begin{abstract}
Summary: The objective of this study was to validate behavioral differences between two groups of drivers through the use of a driving simulator. Controlled experiments in a driving simulator were used to gather objective and subjective evidence on how drivers reacted to roadway objects and handled various hazardous situations. Low-risk, more experienced drivers were more aware of the mental demands of having to remember and later recall a list of items when compared to high-risk, less experienced drivers. Outcomes of the study may potentially serve as the foundation for a training program that will aim to transfer risk assessment strategies from low-risk drivers to high-risk drivers.
\end{abstract}

\section{INTRODUCTION}

With experience drivers learn to better handle their vehicles, and their overall driving skills improve (Lajunen \& Summala, 1995). This experience-driven understanding is considered a form of tacit knowledge that accumulates with experience and is often difficult to verbally articulate (Legree, Heffner, Psotka, Martin, \& Medsker, 2003). According to Sternberg (1997), tacit knowledge is an aspect of practical intelligence that enables individuals to select, shape, and adapt to real-world environments in pursuit of personally valued goals. Practical problems, such as risk assessment in driving, tend to be poorly defined or structured but personally relevant and therefore practical rather than abstract solutions and thinking skills should lead to better acquisition of tacit knowledge.

Several reviews and previous studies have documented the critical importance of risk assessment to driving safety (Deery, 1999; Evans, 1991; Gregersen \& Bjurulf, 1996) and have demonstrated differences between novice and experienced drivers in assessing risks (Trankle, Gelau, \& Metker, 1990), as well as the role of tacit knowledge in risk assessment (Legree, et al., 2003). Through self-reports and subjective surveys, Legree, et al. (2003) showed that more experienced, low-risk drivers are more aware of when and how to modify their driving styles in response to changing conditions. They also found that tacit knowledge (learned through experience) provides more effective assessment information than declarative knowledge (general safety guidelines taught in driving school). It is unclear, however, whether differences in driving behaviors between low-risk and high-risk drivers can be objectively observed.

This study served as an initial step in behaviorally assessing tacit knowledge, specifically to validate behavioral differences between low-risk and high-risk drivers. It was designed to include representative driving situations and to collect objective and subjective reactions to those 
situations in a driving simulator and through self-reports. Two types of distractions (cell phone and passenger) were chosen due to their prevalence in the 100-Car Naturalistic study (Dingus, et al., 2006). The experimental drives included roadway (i.e., driving behind a slow vehicle) and mentally loading (i.e., having a list of groceries in mind to buy) situations considered representative of daily driving experiences that require drivers to constantly assess the level of risk when behind the wheel. It was hypothesized that low-risk drivers would be more thorough in detecting targets (such as pedestrians in certain color shirts) for accomplishing the driving task and more sensitive to various roadway and mental demands when compared to their high-risk counterparts.

\section{METHOD}

\section{Subjects}

Twenty-four participants were recruited through newspaper and electronic mail advertisements to take part in this experiment. Inclusion criteria were as follows: an active driver's license for at least five years, normal or corrected-to-normal vision, normal color vision, normal hearing abilities, native English speaker, and between age 20 and 35 (high-risk group, $n=12, M=26.6$ yrs, $S D=7.3$, average years of driving $=10.4$, average annual mileage $=17,836.8 \mathrm{~km}(11,083.2$ miles)) or between age 40 and 55 (low-risk group, $n=12, M=45.7$ yrs, $S D=4.9$, average years of driving $=29.7$, average annual mileage $=24,140.1 \mathrm{~km}(14,999.9$ miles $)$ ). In addition, the lowrisk drivers were screened for safe driving by not having any major or minor accidents in the past five years. The high-risk drivers were screened for unsafe driving by having multiple minor accidents in the past five years.

The low-risk drivers were older in age and also chosen for demonstrating safe driving histories. High-risk drivers were younger in age and also chosen for demonstrating less safe driving histories. We hoped that by using both age and experience as the selection criteria, the behaviors between the two groups would be more profound and objectively measureable.

Participants were randomly assigned to either the cell phone condition or the passenger condition. There were six participants in each of the condition $\mathrm{x}$ risk level combinations. Participants were compensated for their time at the rate of $\$ 8$ per hour.

\section{Apparatus}

Data were collected at $60 \mathrm{~Hz}$ with a fixed-base, high fidelity driving simulator consisting of a 1998 Saturn SL body and 8-channel $360^{\circ}$ projection screens. The driving scenarios and interactive vehicles were simulated using DriveSafety's Vection ${ }^{\mathrm{TM}}$ Software and its HyperDrive Authoring Suite (Version 1.6.1). Video recordings from three cameras were also collected (driver's face, foot pedals and forward view). A SmartEye Pro, a three-camera, world-referenced infrared eye tracking system from SmartEye $A B$, gathered eye movement data in a non-intrusive manner. 


\section{Tasks}

Driving task. Participants completed one practice drive (approximately 4 minutes) and four experimental drives (approximately 11 minutes), all of which required participants to follow the speed limit of $48.3 \mathrm{~km} / \mathrm{h}$ (30 mph), to maintain the vehicle in the center of the lane, and to drive in a straight, two-lane urban road on a sunny day. In the experimental drives, parked cars and pedestrians were positioned along the road, in the parking lanes and on the sidewalks, respectively. There was a constant flow of ambient traffic in the opposing lane, with a section of it under construction. The driving task mimicked a situation where an employee is running late for work and has about 10 minutes to get there. In two of the experimental drives, participants followed a lead vehicle that drove below the speed limit, with no opportunity to pass.

Another aspect of the driving task required participants to search and detect pedestrians that wore a gray-blue shirt. These target pedestrians were randomly positioned on sidewalks, along with non-target pedestrians that wore white, red, green, brown, and black shirts or dresses. Half of the target pedestrians were walking, and the other half were standing on the sidewalk. Targets were equally likely to be on either side of the road. Figure 1 shows a standing target pedestrian on the left sidewalk.

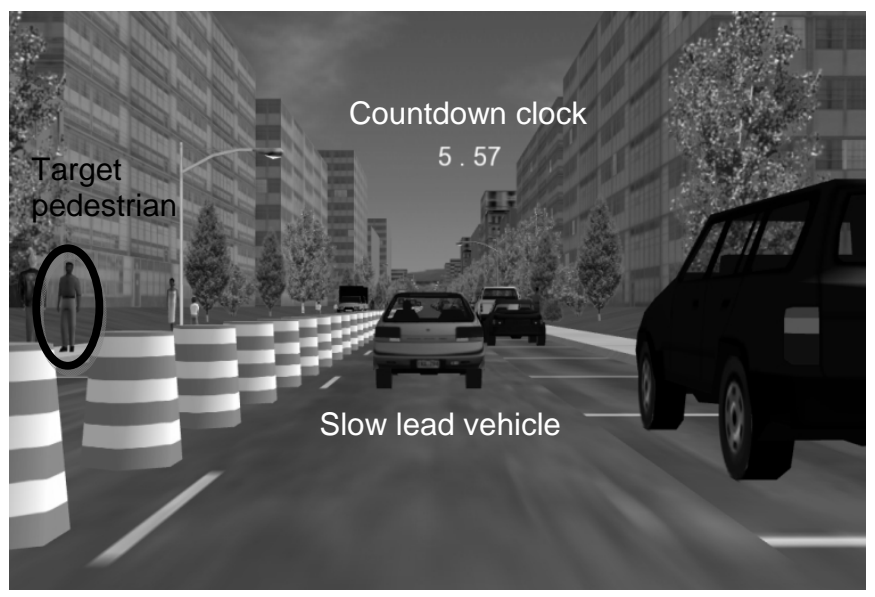

Figure 1. Image from driver's forward view, with a countdown clock, a slow vehicle in front, a target pedestrian on the left sidewalk, parked vehicles, and road construction in the opposing lane

In-vehicle distractions. Voice messages were played during the experimental drives. In two drives, the first voice message (memory call) occurred within two minutes of the drive and contained a total of seven action items the driver needed to complete upon arriving at work. Participants in both cell phone and passenger conditions received the same voice message. Participants needed to remember these action items during the drive and to recall them at the end of the drive. The other three voice messages (distractor call) conveyed a sense of time pressure, and participants did not have to memorize the details of the messages. Each voice message was approximately 50 seconds long and delivered in either voicemail or text message form. 


\section{Procedure}

After participants signed the informed consent, the eye tracker was calibrated for the participants' facial characteristics while seated in the driving simulator. They were asked to make adjustments to the seat and mirrors to suit their size and preference. They were informed of the situation as an employee running late for work on a Monday morning. Participants in the passenger condition were informed that they would be giving a person (sitting in the passenger seat) a ride to work but that the passenger did not wish to talk. Participants were encouraged to get to work within 10 minutes but not told the length of each drive was planned to be longer. If participants did not get to work within 10 minutes, they could continue driving. The clock would then count down to " 0 " and display "time out" on the windshield. When they reached the destination, they were to pull over and park in the parking lane.

Participants drove through a practice drive to become familiarized with the dynamics of the simulator. They were asked to obey traffic laws and drive as they normally do. To ensure that participants understood the instructions, an experimenter sat in the simulator and went through the practice drive with them.

Participants then received instructions to perform the experimental tasks: 1) When a memory call occurs, listen to it and remember its content; 2) For participants in the cell phone condition, press a button in the center console when hearing a ring tone (for memory call and distractor calls) to activate voicemail and listen to the voice messages; For participants in the passenger condition, listen to the messages delivered verbally by the passenger. For drives without a memory call, participants were told to still listen to the three distractor calls but not be required to recall anything at the end of the drives; and 3) Participants should search for pedestrians in gray-blue shirts and press a button on the steering wheel when detection was made. They were also told that they would receive three distractor calls while searching for target pedestrians.

Upon completion of each experimental drive, participants were asked to subjectively rate the level of frustration, mental demand, and time pressure they experienced during the drive and to recall the items from the memory call in two of the drives. The order of the four experimental drives was counterbalanced using a Latin square design.

\section{Experimental Design}

The four experimental drives followed a mixed factorial design with condition (cell phone and passenger) and risk level (low-risk and high-risk) as the between-subject variables and lead vehicle (yes, no) and memory load (yes, no) as the within-subject variables. Data collected during the study session included target detection, percentage of items recalled from the memory call, eye movement, and subjective ratings of frustration, mental demand, and time pressure. Results of the eye movement analysis are not reported here. 


\section{RESULTS}

Repeated measures ANOVAs were used to analyze the effects of risk level, condition, lead vehicle, and memory load on target detection and subjective ratings, and to analyze the effects of risk level, condition, and lead vehicle on percentage of memory recall.

Driving behind a lead vehicle significantly decreased the detection rate from $77.5 \%$ to $72 \%$. $F(1,20)=6.27, p=.0211$. The interaction between condition and lead vehicle was significant, $F(1,20)=5.02, p=.0366$, suggesting that the detection rate was reduced due to the lead vehicle in the cell phone group, $t(20)=3.35, p=.001$, but not in the passenger group. None of the other main effects and interactions reached the significance level.

Driving behind a lead vehicle increased the percentage of items recalled from $48.96 \%$ to $56.77 \%$. $F(1,20)=4.34, p=.05$. There was a significant interaction between condition and lead vehicle, $F(1,20)=5.58, p=.0284$, indicating that the percentage of items recalled increased in the presence of the lead vehicle for the passenger group but remained the same with or without the lead vehicle for the cell phone group. None of the other main effects or interaction effects reached the significance level.

The presence of a lead vehicle increased the level of perceived frustration, $F(1,19)=19.71, p=$ .0003 , from 2.31 to 3.15 . The interaction between condition and memory load was significant, $F(1,19)=4.42, p=.049$. Memory load increased the rating of frustration in the passenger group but not in the cell phone group, $t(19)=2.81, p=.006, t(19)=0.24, p=.814$, respectively. None of the other main effects or interaction effects reached the significance level.

The presence of a lead vehicle increased the level of perceived mental demand, $F(1,19)=5.45, p$ $=.0306$, from 2.72 to 3.13. Memory load also increased the level of perceived mental demand, $F(1,19)=11.95, p=.0026$, from 2.63 to 3.23 . The interaction between risk level and memory load was significant, $F(1,19)=4.10, p=.047$, indicating that memory load imposed a significant increase of mental demand on low-risk drivers but not on high-risk drivers, $t(19)=$ $3.74, p=.003, t(19)=1.05, p=.801$, respectively. None of the other main effects or interaction effects reached the significance level.

The interaction between condition and memory load on time pressure was significant, $F(1,19)=$ $5.05, p=.0367$, indicating that memory load had opposite effects on perceived time pressure for the two conditions of participants. For the cell phone condition, perceived time pressure was lower with a memory call than without a memory call. For the passenger condition, perceived time pressure was greater with a memory call than without one. None of the main effects or other interaction effects reached the significance level.

\section{DISCUSSION}

Overall, participants were sensitive to the experimental manipulations, validating that they did introduce additional demands on drivers, especially the presence of a slow lead vehicle and a passenger. Low-risk participants were relatively more sensitive to the additional mental demand than their high-risk counterparts. 
Results did not support the hypothesis that low-risk, safer drivers would have more thorough detection of targets. There are at least two possible explanations. First, the sample size was small, and the selection criteria might not have been ideal. Participants in the high-risk group all had multiple accidents and violations with an average of 10 years driving experience. Arguably, they are moderately experienced drivers. Therefore, their behaviors might not be representative of unsafe or less safe drivers. Age and driving history may not be sufficient indicators for distinguishing safe and unsafe drivers. Perhaps a better recruiting approach would be to invite professional drivers and driving instructors, while keeping their driving history and age as covariates. A larger sample size with balanced age and group dividends would also improve the generalizability of findings.

Second, participants commented that not being able to talk to the passenger (who was supposed to be a friend or co-worker) was awkward. This artificial manipulation might have altered participants' strategies and overall perception of the driving situation. It seemed likely that having a stranger in the car made the participants invest more effort but also introduced some level of discomfort. Participants felt frustrated when they could not remember more items and feared they were being watched instead of merely giving a person a ride to work. Although the passenger condition was somewhat artificial, it was a necessary control for having the same content and duration of messages in both cell phone and passenger conditions and for eliminating any effect due to interactive conversations in the passenger group.

The current study was unique in that it implemented representative driving experiences (e.g., construction zone, cars in front driving below speed limit, a to-do list to remember, being late for work), and the majority of the participants commented that they felt the scenario was close to what they have experienced and would normally do in real life. The generalizability of results from the simulator context to the real world is somewhat constrained due to the abovementioned limitations. However, the results suggest that the simulator can be used to create increased hazard across groups so experience-based strategies in assessing and mitigating this risk can be exposed and studied. It is expected that with better recruiting and selection of participants, behavioral and strategic differences between safe and unsafe drivers can be better observed from scenario-based driving environments.

\section{ACKNOWLEDGMENT}

This work was supported by a Phase I Small Business Technology Transfer (STTR) Program from the U.S. Army Research Institute. The authors would like to acknowledge the contributions of the research programmers and assistants, Ron Carbonari, Lisa Skinner, Henry Chen, and Sara Butler.

\section{REFERENCES}

Deery, H. A. (1999). Hazard and risk perception among young novice drivers. Journal of Safety Research, 30(4), 225-236. 
Dingus, T. A., Klauer, S. G., Neale, V. L., Petersen, A., Lee, S. E., Sudweeks, J., et al. (2006). The 100-Car naturalistic driving study, phase II - Results of the 100-Car field experiment. From http://www-nrd.nhtsa.dot.gov/departments/nrd-13/driverdistraction/PDF/100CarMain.pdf

Evans, L. (1991). Traffic safety and the driver. New York: Van Nostrand Reinhold.

Gregersen, N. P., \& Bjurulf, P. (1996). Young novice drivers: Towards a model of their accident involvement. Accident Analysis and Prevention, 28(2), 229-241.

Lajunen, T., \& Summala, H. (1995). Driving experience, personality, and skill and safety-motive dimensions in drivers' self-assessments. Personality and Individual Differences, 19(3), 307318.

Legree, P. J., Heffner, T. S., Psotka, J., Martin, D. E., \& Medsker, G. J. (2003). Traffic crash involvement: Experiential driving knowledge and stressful contextual antecedents. Journal of Applied Psychology, 88(1), 15-26.

Sternberg, R. J. (1997). Successful intelligence. New York: Plume Books.

Trankle, U., Gelau, C., \& Metker, T. (1990). Risk Perception and Age-Specific Accidents of Young Drivers. Accident Analysis and Prevention, 22(2), 119-125. 